\title{
Planejamento de recursos humanos de enfermagem: desafio para as lideranças
}

\author{
Planning human resources in nursing: challenge for the leadership \\ Planificación de recursos humanos en enfermería: desafío para el liderazgo
}

\author{
Ana Maria Muller de Magalhães', Caren de Oliveira Riboldi', Clarice Maria Dall'Agnol' \\ 'Universidade Federal do Rio Grande do Sul. Escola de Enfermagem. \\ Núcleo de Estudos sobre Gestão em Enfermagem. Porto Alegre, RS
}

Submissão: 20/08/2008

Aprovação: 15/06/2009

\begin{abstract}
RESUMO
Propõe-se um ensaio das Questões Que envolvem o planejamento de recursos humanos de enfermagem na área hospitalar em nossa realidade, abordando possíveis temas para futuras pesquisas. Traça-se uma retrospectiva da evolução das pesQuisas sobre dimensionamento de pessoal no país e a incorporação de novos instrumentos para avaliação da carga de trabalho com base no grau de dependência dos pacientes e nas ações de cuidado. Discute-se o impacto do Quantitativo e Qualitativo de recursos humanos nos resultados assistenciais e o papel das lideranças na adequação do Quadro de pessoal, com vistas a fornecer um atendimento seguro, livre de riscos aos pacientes e suas famílias, buscando um modelo de gestão das práticas de saúde na perspectiva do cuidado complexo.
\end{abstract}

Descritores: Carga de trabalho; Recursos humanos de enfermagem; Cuidados de enfermagem/organização e administração.

\section{ABSTRACT}

This study proposes an essay of issues involving human resources planning in nursing in the hospital sector in our current reality, discussing possible topics for future research. This is a retrospective of the evolution of research on determining the number of staff in Brazil, and the incorporation of new instruments to evaluate the work load based on the degree of dependency of the patients and actions in care. The impact of the Quantity and Quality of human resources on the results of care is discussed, and the role of leaders in adapting the staff composition, in order to supply safe, risk-free care to the patients and their families, seeking a management model for health practices from the perspective of complex care.

Descriptors: Work load; Nursing staff; Nursing care/organization and administration.

\section{RESUMEN}

Se propone un ensayo sobre la etapa del desarrollo de las inquietudes Que involucran la planificación de recursos humanos de enfermería en el área hospitalaria en nuestra realidad, abordando posibles temas para futuras investigaciones. Se traza una retrospectiva de la evolución de las investigaciones sobre dimensionamiento de personal en nuestro país y la incorporación de nuevos instrumentos para evaluación de la carga de trabajo con base en el grado de dependencia de los pacientes y en las acciones de cuidado de salud. Se discute sobre el impacto de lo cuantitativo y cualitativo de recursos humanos en los resultados asistenciales y el papel de los liderazgos en la adecuación del cuadro de personal, con el objetivo de suministrar una atención segura, libre de riesgos a los pacientes y sus familias, buscando un modelo de gestión de las prácticas de salud en la perspectiva del cuidado complejo.

Descriptores: Carga de trabajo; Recursos humanos de enfermería; Cuidados de enfermería/organización y administración. 


\section{INTRODUÇÃO}

As Questões Que envolvem a gestão em saúde e em enfermagem estão cada vez mais presentes no cotidiano de gestores, profissionais da área e pesquisadores. A complexidade crescente do processo de produção de cuidados exige a reestruturação dos modelos de gestão e das organizações para fornecer respostas aos desafios da demanda de atendimento no Sistema Único de Saúde (SUS).

A prestação de serviços de saúde, diferente de outros setores produtivos, ainda não conseguiu substituir os recursos humanos por máQuinas ou robôs Que possam assegurar o atendimento ao paciente. O advento de várias tecnologias tem auxiliado no monitoramento mais rigoroso das condições clínicas do paciente, assim como na precisão das intervenções terapêuticas, gerando a necessidade de maior Qualificação dos profissionais para sua incorporação sem causar impacto na diminuição de trabalhadores necessários para operá-las.

Quando se discute aspectos Que envolvem o processo de trabalho em saúde e as demandas da população, torna-se imprescindível abordar temas referentes ao dimensionamento de pessoal, carga de trabalho, condições de trabalho e absenteísmo, entre outros aspectos Que interferem na produção de cuidados. Os estudos sobre recursos humanos em saúde e na enfermagem remontam à década de 60, mas somente nos anos 80 ganham impulso debatendo acerca da formação profissional, organização social das práticas/trabalho em saúde, força de trabalho em enfermagem (FTE) e mercado de trabalho ${ }^{(1)}$.

O panorama mundial aponta dificuldades para as Questões de saúde Que envolvem as enormes diferenças entre os países desenvolvidos e os países pobres. O cenário de saúde no Brasil também apresenta vários contrastes e desafios a serem superados mediante problemas estruturais, políticos e culturais de organizações e sistemas complexos como o SUS. Na área de enfermagem, revelase uma escassez de mão-de-obra, carência de Qualificação profissional, excessiva carga de trabalho e evasão da profissão, ocasionando um fenômeno de migração de profissionais para os países ricos ${ }^{(2)}$.

Em nosso meio, estudos relacionados com a carga de trabalho da enfermagem, grau de dependência dos pacientes e dimensionamento de pessoal de enfermagem têm sido liderados por Gaidzinski ${ }^{(3)}$, a Qual desenvolveu um método específico para cálculo de pessoal, aprimorando-o no sentido de adequá-lo à realidade brasileira. Este modelo define as etapas de identificação da carga média de trabalho diária na assistência e determina a proporção ideal das categorias profissionais de enfermagem, considerando a jornada de trabalho da equipe de enfermagem e o Índice de Segurança Técnica.

Acompanhando as discussões e a produção científica relacionada ao tema de dimensionamento de pessoal em enfermagem, percebese a evolução das pesquisas e a ampliação de sua abrangência para além da estimativa de cálculo de pessoal, incorporando aspectos como o impacto nos custos da saúde e resultados assistenciais, o estudo do grau de dependência dos pacientes nos cuidados de enfermagem, a carga de trabalho da equipe de enfermagem e os fatores Que interferem na produtividade dos profissionais, relacionados às condições de trabalho e à saúde do trabalhador, entre outros.
A problemática em pauta consiste num tema recente nas pesquisas acadêmicas, com lacunas de conhecimento e com repercussões ainda modestas na prática, pois a falta de recursos humanos de enfermagem nas organizações de saúde é uma afirmação constante de enfermeiros e gestores responsáveis pelo atendimento. Assim, propõe-se uma reflexão e análise sobre o estágio do desenvolvimento das Questões Que envolvem o planejamento de recursos humanos de enfermagem em nossa realidade, subsidiando a elaboração de futuras pesquisas.

\section{DIMENSIONAMENTO DE RECURSOS HUMANOS DE ENFERMAGEM E A CARGA DE TRABALHO}

O primeiro método de planejamento de recursos humanos em enfermagem surgiu em torno do século XVII, com Florence Nightingale, pioneira da administração hospitalar e precursora da profissão de enfermagem. Denominado intuitivo, baseava-se na subjetividade e na relação proporcional entre trabalhadores e tarefas, considerando a gravidade dos pacientes.

Atualmente, devido à complexidade dos cuidados e do processo de trabalho em saúde tem se buscado desenvolver modelos de adequação de pessoal de enfermagem de acordo com as características dos pacientes e grau de dependência dos cuidados de enfermagem. O dimensionamento de pessoal tem sido considerado um desafio, haja vista Que estes recursos são os mais complexos da organização, e os demais recursos exigem a sua presença, para Que possam ser utilizados ${ }^{(4)}$.

Apesar de ter havido algumas iniciativas de classificar os pacientes Quanto ao grau de dependência e o estabelecimento de horas de enfermagem nas décadas de $70-80^{(5-7)}$, apenas na última década os órgãos oficiais da enfermagem e saúde no Brasil têm divulgado critérios e parâmetros Que orientem o planejamento de recursos humanos em enfermagem nas organizações de saúde. As Resoluções COFEN no $189 / 96$ e 293/2004(8,9) estabeleceram os primeiros parâmetros oficiais para o dimensionamento de pessoal de enfermagem nas instituições de saúde e assemelhados, definindo o Quanti-Qualitativo mínimo nos diferentes níveis de formação para a cobertura assistencial.

Tais iniciativas, ainda recentes, contribuem com os enfermeiros da prática na busca de solução para os problemas cotidianos de adeQuação de pessoal em um contexto onde a demanda, na maioria das vezes, é maior do que a oferta de atendimento, implicando em riscos para os pacientes. A implementação das políticas de saúde e da filosofia de trabalho da enfermagem está diretamente relacionada com a Qualidade e a Quantidade dos profissionais Que irão executá-las.

No hospital, "o processo de cuidar, como eixo central do trabalho em enfermagem, se constitui num processo complexo contínuo e Que não pode ser adiado ou interrompido em várias circunstâncias", denotando a necessidade de um adequado planejamento de recursos humanos ${ }^{(10)}$. Neste contexto, é importante salientar a tendência de atribuir os altos custos da saúde aos gastos com o Quadro de pessoal, entretanto, o processo de atendimento em saúde é fundamentalmente uma relação de pessoas Que implica em interação, intervenção e trocas. A resolutividade e a Qualidade do atendimento em saúde estão intrinsecamente relacionadas aos recursos humanos Que os desenvolvem.

Em vários estudos o dimensionamento de pessoal tem sido foco 
de atenção das enfermeiras e gestores dos serviços de saúde por interferir diretamente na eficácia e no custo do atendimento a saúde, contudo, a falta de parâmetros para o planejamento e avaliação de recursos humanos tem levado as lideranças de enfermagem a equacionar pessoal de forma empírica, baseadas na experiência e no julgamento intuitivo ${ }^{(11-13)}$.

Inúmeros pesquisadores no Brasi $^{(1,14-17)}$ buscaram em modelos internacionais (escalas e escores de gravidade) possibilidades de testar na realidade brasileira parâmetros para estimar o tempo despendido no cuidado ao paciente. Outros autores ${ }^{(4,18,19)}$ elaboraram modelos e escalas para classificar os pacientes de acordo com o grau de dependência, traduzidos em valores numéricos Que compõem as variáveis Quantitativas nas fórmulas para medir o tempo despendido pela equipe de enfermagem no cuidado a um grupo de pacientes e determinar o número de profissionais necessários. Considerando as especificidades da área e os custos envolvidos, muitos destes estudos estão centrados na área de terapia intensiva, porém já existem iniciativas de aplicar estes métodos em outros espaços hospitalares, buscando aproximar os modelos teóricos da realidade com vistas a dar respostas à necessidade de alocar pessoal adequado para cada setor de atendimento.

Estas considerações nos remetem a reflexões acerca do tema de dimensionamento de pessoal na realidade brasileira, onde já existem modelos capazes de avaliar e comparar o Quadro de pessoal existente e o projetado como adequado, a partir da contextualização e especificação de diferentes cenários, como terapia intensiva e unidades de internação. $\mathrm{O}$ Que se observa nestes estudos são os valores atribuídos às horas de enfermagem por tipo de paciente, sem Que haja melhor definição e especificação acerca destes parâmetros. Dessa forma, Questiona-se sobre a acurácia e aplicabilidade nos diferentes cenários brasileiros. Na prática, a aplicação destes modelos tem gerado dificuldades na adeQuação do número de pessoal, ficando o sentimento da equipe de enfermagem Que estes cálculos são subestimados e Que as escalas não retratam a verdadeira realidade de trabalho.

A incorporação do termo carga de trabalho aos estudos de dimensionamento de pessoal de enfermagem ocorre no sentido de estimar a demanda de trabalho requerida dos profissionais de enfermagem nas atividades de cuidado direto e indireto ao paciente. A carga de trabalho é medida ou expressa em escores ou pontuações Que determinam o número de horas dedicadas pela equipe de enfermagem a cada paciente. Devido ao emprego do conceito de carga de trabalho com este sentido, é importante fazer uma distinção do termo em outras áreas do conhecimento.

O conceito de carga de trabalho é amplamente utilizado no campo da ergonomia, saúde do trabalhador e ciências sociais, principalmente na perspectiva do materialismo histórico, buscando dar sentido e compreender os fatores envolvidos no processo de trabalho e seu impacto sobre o trabalhador. Neste caso, o termo carga de trabalho diz respeito ao conjunto de exigências físicas (carga física do trabalho - CFT) e psíquicas (carga psíquica ou emocional - CP/CE) necessárias para a execução de uma tarefa, podendo ser utilizado referindo-se exclusivamente à tarefa ou simultaneamente à tarefa e ao envolvimento físico e social ${ }^{(20,21)}$.

Nessa perspectiva, inicialmente, os estudos empregaram instrumentos para medir o esforço físico empenhado pelo trabalhador na execução de tarefas específicas procurando avaliar os impactos fisiológicos e desgaste físico, numa abordagem mais clássica e reducionista do processo de trabalho. Posteriormente, incorporou-se a dimensão de carga mental (desgaste psíquico ou emocional) necessária à execução do trabalho, buscando maior abrangência de compreensão não apenas sobre a tarefa, mas sobre o envolvimento e significado Que o trabalhador atribui ao seu trabalho ${ }^{(21)}$. Atualmente, devido à importância do mundo do trabalho em nossa sociedade, estuda-se as dimensões físicas, psicológicas e sociais envolvidas no processo de trabalho.

Uma importante Questão Que pode ser problematizada nos instrumentos de medida da carga de trabalho para a equipe de enfermagem é que estes se focam nas dimensões das dependências físicas, fisiológicas e terapêuticas do paciente, numa forte influência do modelo clínico de assistência à saúde, centrado na doença, caracterizado por uma centralização no atendimento médico individual e curativo, com foco na terapêutica médica ${ }^{(22,23)}$. Talvez isto possa explicar a percepção das equipes de enfermagem de Que os pacientes requerem mais cuidados do que é retratado por estas escalas.

Outro fator Que merece destaque nesta discussão é o papel da equipe de enfermagem na gestão do cuidado de saúde Que, através de políticas de humanização e diretrizes do SUS, tem garantido melhor acesso e acompanhamento dos pacientes e seus familiares na área hospitalar. Esta mudança se reflete na organização do trabalho da equipe de enfermagem para as ações de cuidado, assim como avança no sentido de assegurar a autonomia e participação junto aos usuários dos serviços de saúde. Alguns instrumentos de medida da carga de trabalho já contemplam o investimento da equipe de enfermagem em ações de orientação e educação para o paciente e sua família.

O pressuposto para uma nova gestão das práticas de saúde está intrinsecamente relacionado com os modos como se organiza o processo de cuidado nos serviços de saúde. A enfermagem, em sua formação e prática, visa a integralidade das ações assistenciais com toda a complexidade Que perpassa o ser cuidado, no entanto, observa-se no cotidiano de trabalho, Que a demanda de tarefas e o grande número de pacientes Que necessitam de intervenções dificultam a interação profissional/paciente/família. Trabalhar no sentido de modificar as condições de trabalho e práticas de saúde passa a ser um imperativo dos profissionais e atores sociais Que vivenciam este processo.

\section{IMPACTO DOS RECURSOS HUMANOS NA QUALIDADE DA ASSISTÊNCIA E O COMPROMISSO DAS LIDERANÇAS}

Mesmo reconhecendo a importância dos recursos humanos na Qualidade da assistência, as lideranças de enfermagem encontram enormes resistências para adequar o número de pessoal à demanda de atendimento nas instituições de saúde, principalmente em razão de justificativas orçamentárias.

Estudos internacionais ${ }^{(24,25)}$ têm buscado avaliar o impacto da Quantidade e Qualificação dos profissionais de enfermagem nos resultados assistenciais dos pacientes, destacando-se a escassez de pessoal e a necessidade do desenvolvimento de políticas educacionais e profissionais para tornar este campo de trabalho mais atraente e retentor, ou seja, com menor rotatividade de pessoal.

A relação da equipe de enfermagem com a segurança dos 
pacientes e o impacto nos resultados da assistência tem sido foco de discussão e trazem dados preocupantes da realidade americana e européia, Quando afirmam Que o aumento de um paciente por enfermeiro aumenta a probabilidade de $7 \%$ na mortalidade de pacientes cirúrgicos ou Que o aumento de um paciente por enfermeiro aumenta a probabilidade de $23 \%$ na insatisfação no trabalho pelo enfermeiro e de $15 \%$ de chance do mesmo apresentar Burnout (síndrome Que se caracteriza por sintomas de exaustão no trabalho) ${ }^{(24)}$.

Devido à complexidade, gravidade e altos custos nas áreas de terapia intensiva, muitos estudos têm se desenvolvido nesta área para avaliar a carga de trabalho de enfermagem, tanto para estimar o Quantitativo de pessoal, como para auxiliar no planejamento orçamentário. Um dos instrumentos bastante utilizados dentro dessa proposta é o Nursing Activitie Score (NAS), Que visa Quantificar os cuidados de enfermagem e o grau de complexidade das atividades junto aos pacientes durante um turno de trabalho(17).

Enquanto estudos internacionais apontam os riscos da inadeQuação de pessoal, gerando sobrecarga de trabalho e falhas no cuidado ao paciente, há poucos estudos no Brasil aprofundando a temática. É importante para a realidade brasileira, confirmar os pressupostos levantados de Que o número de horas de enfermagem está diretamente relacionado com a segurança dos pacientes e Que melhores níveis no Quadro de enfermagem resultam num decréscimo da média de permanência no hospital, diminuição das taxas infecção urinária, pneumonia e choque ${ }^{(2)}$.

Um recente estudo nacional, apresentou resultados Que convergem com os achados internacionais, indicando Que os trabalhadores de enfermagem apresentam absenteísmo relacionado às doenças provenientes do cansaço físico e mental, pelas más condições de trabalho, à jornada diária e a realização de tarefas em ritmo acelerado(26).

A partir destas considerações e do avanço de alguns estudos na área de planejamento de recursos humanos em enfermagem, não se pode adotar postura omissa frente aos riscos a Que estão submetidos os pacientes diante de um Quadro de pessoal inadequado para desenvolver as ações de cuidado e de saúde. O compromisso das lideranças de enfermagem com a adequação do Quadro de pessoal e seu impacto na organização do trabalho em saúde é crucial para o alcance de um cuidado individualizado, integral e seguro.

Quando se estabelecem relações entre dimensionamento de pessoal, o papel das lideranças na condução deste processo e a segurança do paciente, amplia-se a discussão para um dos temas mais atuais no cenário de saúde, Que tem mobilizado recursos da Organização Mundial de Saúde ${ }^{(27)}$ e das organizações de enfermagem no mundo todo, lançando políticas e programas para identificar e atenuar os riscos aos pacientes internados. Todas as ponderações apresentadas sinalizam a complexidade do tema e o desafio que temos pela frente.

\section{CONSIDERAÇÕES FINAIS}

As constantes transformações e avanços nos cenários de produção de práticas de saúde, especialmente no ambiente hospitalar, têm repercutido nas formas como as equipes se organizam para prestar atendimento de saúde. A mudança da concepção de paciente, como um indivíduo passivo, para usuário de saúde e a presença da família dentro dos hospitais, socializam o conhecimento das práticas de cuidado e das dificuldades enfrentadas pelos serviços de saúde. Pode-se dizer Que esta inclusão tem auxiliado os profissionais de saúde a repensar as suas práticas e modos de cuidar.

Mesmo enfrentando resistências, baseadas num modelo de saúde marcado pela hegemonia médica, a enfermagem desempenha um papel central nos sistemas de saúde e no processo de atendimento ao paciente, sendo esta uma condição essencial para a transformação de realidades. Em tal cenário, destaca-se a fundamental importância das lideranças de enfermagem na busca de soluções e novos modelos de gestão Que respondam às dificuldades de alocação de recursos humanos, tecnológicos e financeiros, assegurando um processo de atendimento baseado nas melhores práticas e Que garanta a segurança dos pacientes.

A tentativa de descrever, compreender e analisar os fatores envolvidos no processo de trabalho de enfermagem para além da dimensão técnica ou explícita do cuidado ao paciente e sua família, implica em encontrar formas de avaliar além do tempo medido na execução das tarefas e dar corpo às outras dimensões do cuidado em saúde Que exigem grande investimento dos profissionais, seja em desgaste físico (horas de trabalho) como em desgaste mental ou emocional para lidar com as situações Que envolvem as interações e intervenções no processo de trabalho em saúde.

A gestão das práticas de saúde na perspectiva da complexidade parece trazer um caminho para discussão dos modelos utilizados até então, apontando para a necessidade de resgatar o paradigma do humano na organização do trabalho em saúde e em enfermagem. A partir destas considerações, levanta-se a possibilidade de buscar modelos de avaliação e análise da carga de trabalho de enfermagem, Que tenham a sensibilidade de incluir as dimensões mais subjetivas das ações de cuidado com o paciente e sua família.

Os estudos analisados apontam a necessidade de aprofundar o tema em Questão, ampliando o foco de entendimento sobre as variáveis Que interferem no planejamento de recursos humanos de enfermagem, considerando toda a complexidade e especificidade do trabalho nas organizações de saúde. Os avanços deste campo de conhecimento são fundamentais para subsidiar as lideranças de enfermagem na tomada de decisões com vistas a assegurar um cuidado integral, individualizado e humano, livre de riscos aos pacientes.

\section{REFERÊNCIAS}

I. Silva LIMC, Peduzzi M. Os recursos humanos de enfermagem na perspectiva da força de trabalho: análise da produção científica. Rev Esc Enferm USP 2005; 39(esp): 589-96.

2. Chagurutu S, Vallabhameni BA. Aiding and abetting-nursing crises at home and abroad. N Engl I Med 2005; 353(17): 176 I 1763.

3. Gaidzinski RR. O dimensionamento de pessoal de enfermagem em instituições hospitalares [tese]. São Paulo: Escola de Enfermagem, Universidade de São Paulo; 1998.

4. Gaidzinski RR. Dimensionamento de pessoal de enfermagem. In: Kurcgant P, organizadora. Administração em Enfermagem. São Paulo: EPU; 1991. p.91-96. 
5. Ribeiro CM. Sistema de classificação de pacientes como subsídio para movimento de pessoal de enfermagem [tese]. São Paulo: Escola de Enfermagem, Universidade de São Paulo; 1972.

6. Alcalá MU, Nunes MF, Kato T, Reigada I, Silva RL, Yoshimura DK. Cálculo de pessoal: estudo preliminar para o estabelecimento de euadro de pessoal de enfermagem na superintendência médico hospitalar de urgência. São Paulo: Secretaria de Higiene e Saúde; 1982.

7. Campedelli MC, Taketo C, Sancinetti TR, Benko MA. Cálculo de pessoal de enfermagem: competência da enfermeira. Rev Esc Enf USP 1987; 2 I(1): 3-15.

8. Conselho Federal de Enfermagem (BR). Resolução COFEN- I 89/ 1996, de 25 de março de 1996. Estabelece parâmetros para dimensionamento do Quadro de profissionais de Enfermagem nas instituições de saúde. Porto Alegre: COREN/RS; 1996. [citado em: 20 nov 2008]. Disponível em: URL: http:// www.portalcoren-rs.gov.br/web/resoluca/r l 89.htm.

9. Conselho Federal de Enfermagem (BR). Resolução COFEN 293/ 2004, de 2 I de setembro de 2004. Fixa e estabelece parâmetros para o dimensionamento do Quadro de profissionais de Enfermagem nas unidades assistenciais das instituições de saúde e assemelhados. Porto Alegre: COREN/RS; 2004. [citado em: 20 nov 2008]. Disponível em: URL: http://www.portalcorenrs.gov.br/web/resoluca/r293.htm.

10. Echer IC, Moura GM, Magalhães AM, Piovesan R. Estudo do absenteísmo como variável no planejamento de recursos humanos em enfermagem. Rev Gaúcha Enferm 1999; 20(2): $65-76$.

11. Gaidzinski RR. O dimensionamento de pessoal de enfermagem segundo a percepção de enfermeiros Que vivenciam esta prática [tese]. São Paulo: Escola de Enfermagem, Universidade de São Paulo; 1994.

12. Magalhães AMM, Duarte ERM, Moura GMSS. Estudo das variáveis Que participam do dimensionamento de pessoal de enfermagem em hospitais de grande porte. Rev Gaúcha Enferm 1995; 16(1/2): 5-16.

13. Gaidzinski RR, Fugulin FMT, Castilho V. Dimensionamento de pessoal de enfermagem em instituições de saúde. In: Kurcgant P, organizadora. Gerenciamento em enfermagem. Rio de Janeiro: Guanabara Koogan; 2005. p.125-137.

14. Conishi RMY, Gaidzinski RR. Nursing activities score (NAS) como instrumento para medir a carga de trabalho de enfermagem em UTI adulto. Rev Esc Enferm USP 2007;4I (3): 346-54.
15. Padilha KG, Souza RMC, Miyadahira AMK, Cruz DALM, Vattimo MFF, Kimura M, et al. Therapeutic intervention scoring system28 (TISS 28): diretrizes para aplicação. Rev Esc Enferm USP 2005; 39(2): 229-33.

16. Gonçalves LA, Padilha KG. Fatores associados à carga de trabalho de enfermagem em Unidade de Terapia Intensiva. Rev Esc Enferm USP 2007;4I (4): 645-52.

17. Queijo AF. Tradução para o português e validação de um instrumento de medida da carga de trabalho de enfermagem em Unidade de Terapia Intensiva: Nursing Active Score (N.A.S.) [dissertação]. São Paulo: Escola de Enfermagem, Universidade de São Paulo; 2002.

18. Perroca MG. Instrumento de classificação de pacientes de Perroca: validação clínica [tese]. São Paulo: Escola de Enfermagem, Universidade de São Paulo; 2000.

19. Fugulin FMT. Dimensionamento de pessoal de enfermagem: avaliação do Quadro de pessoal das unidades de internação de um hospital de ensino [tese]. São Paulo: Escola de Enfermagem, Universidade de São Paulo; 2002.

20. Associação Portuguesa de Ergonomia. Apergo. Conceitos. [citado em: 10 dez 2008]. Disponível em: URL: http:// www.apergo.pt/ergonomia/conceitos.php

21. Dejours C, Dessors D, Desrlarux F. Por um trabalho, fator de equilíbrio. Rev Adm Empresas 1993; 33(3): 98- 104.

22. Merhy EE, Onocko R, organizadores. Agir em saúde: um desafio para o público. São Paulo: HUCITEC; 2002.

23. Gonçalves RBM. Tecnologia e organização social das práticas de saúde: características tecnológicas do processo de trabalho na rede estadual de centros de saúde de São Paulo. São Paulo: HUCITEC; 1994.

24. Aiken L, Clarke S, Sloane DM, Sochalski J, Silber JH. Hospital nurse staffing and patient mortality, nurse burnout and job dissatisfaction. JAMA 2002; 288(16): 1987-93.

25. Aiken LH, Clarke SP, Cheung RB, Sloane DM, Silber JH. Educational levels of hospital nurses and surgical patient mortality. IAMA 2003; 290(12): 1617-23.

26. Costa FM, Vieira MA, Sena RR. Absenteísmo relacionado à doenças entre membros da equipe de enfermagem de um hospital escola. Rev Bras Enferm 2009; 62(I): 38-44.

27. World Heatlh Organization. World alliance for patient safety. Washington DC (USA); 2004. [citado em: 15 nov 2007]. Disponível em: URL: http://www.who.int/patientsafety/en/ 